\title{
The effect of BM67 gene deletion on Bombyx mori nuclear polyhedrosis virus replication
}

\author{
Y. SHI ${ }^{1,2}$, C. ZHANG ${ }^{1,2}$, C. XIE ${ }^{1,2}$, Y. QUAN ${ }^{1,2}$, Z. NIE ${ }^{1,2}$, J. CHEN ${ }^{1,2}, Z^{2}$ LV $^{1,2}$, Y. ZHANG ${ }^{1,2}$, W. YU ${ }^{1,2^{*}}$ \\ ${ }^{1}$ College of life sciences, Zhejiang Sci-Tech University, Zhejiang Province, Hangzhou, P. R. China; ${ }^{2}$ Zhejiang Provincial Key Laboratory \\ of Silkworm Bioreactor and Biomedicine, Zhejiang Province, Hangzhou, P. R. China
}

Received May 6, 2014; accepted February 10, 2015

\begin{abstract}
Summary. - Homologs of Bombyx mori nuclear polyhedrosis virus (BmNPV) Bm67 gene ORF67 have been found in the genome of all lepidopteran nuclear polyhedrosis viruses, but their function is still not very clear. In order to analyze it we employed a bacmid harboring the complete BmNPV genome including the Bm67 gene and expressing infectious virus (wtBacmid) for the construction of its Bm67-deficient variant (Bm67-KO-Bacmid) using the Red recombination system and the Bm67-repaired variant (Bm67-Re-Bacmid) using the Bac-to-Bac system. By transfecting BmN cells with these bacmids we demonstrated that the Bm67deficient virus did not generate infectious virus, while the repaired virus restored its infectivity, indicating that the $B m 67$ gene is essential for the formation of infectious budding virus (BV). Electron microscopy of BmN cells transfected with the abovementioned bacmids showed many mature rodshaped virus particles in both wtBacmid- and Bm67-Re-Bacmid-transfected cells but none in Bm67-KO-Bacmid-transfected ones. Moreover, the real-time RT-PCR showed that the deletion of $B m 67$ from wtBacmid significantly reduced the levels of viral genomic DNA and transcripts of viral early genes dnapol, ie-1 and lef- 3 but not those of transcripts of late gene $v p 39$ and very late gene $p 10$. The finding that the Bm67-deficient virus generated reduced levels of infectious virus and transcripts of early dnapol gene but not those of late genes indicates that the $B m 67$ gene is essential for BmNPV replication.
\end{abstract}

Keywords: Bombyx mori nuclear polyhedrosis virus; Bm67 gene; virus replication; genome replication; genome transcription

\section{Introduction}

Baculoviridae comprise the largest known group of insect viruses. Baculoviruses are specialized pathogenic microorganisms parasitic to arthropods, and their hosts are mainly among the Lepidoptera, Diptera, Hymenoptera, Coleoptera, and Trichoptera of the class Insecta. According to

*Corresponding author. E-mail: mikkyu@163.com; phone: +86571-86843190. Postal address: Xiasha High-Tech Zone No.2 Road, Hangzhou 310018, P. R. China.

Abbreviations: AcMNPV = Autographa californica multiple nuclear polyhedrosis virus; $\mathrm{BmNPV}=$ Bombyx mori nuclear polyhedrosis virus; $\mathrm{BV}=$ budded virus; ORFs = open reading frames; p.t. $=$ post transfection the morphological characteristics of baculovirus infection, including the presence of inclusion bodies, baculoviruses can be divided into two genera, Granulovirus and Nucleopolyhedrovirus (Bulach et al., 1999).

The first baculovirus genome fully sequenced was Autographa californica multiple nuclear polyhedrosis virus (AcMNPV) (Ayres et al., 1994). Its genome is composed of 133,894 bases with 154 open reading frames (ORFs) encoding 150 or more genes. As of 2009, 47 different baculovirus genomes have been sequenced, ranging in size from 80 to $180 \mathrm{~kb}$, in which $90 \%$ of the genes encode proteins and very few genes overlap. The genome of the T3 strain Bombyx mori nuclear polyhedrosis virus (BmNPV) was sequenced by Gomi et al. (1999), and is 128,413 bp in length, with 135 ORFs, each encoding 60 or more amino-acid residues. Com- 
parative genomic studies suggest that the genome is similar in structure to AcMNPV, with 115 highly conserved ORFs with greater than $90 \%$ homology (Yu et al., 2013).

AcMNPV, the model species for baculoviruses infecting Lepidoptera, is the most studied baculovirus. AcMNPV gene expression is strictly regulated at the transcriptional level, and AcMNPV genes can be divided into those expressed in the early, late, and very late stages of viral replication (Passarelli et al., 2007). Early gene expression is regulated by the host RNA polymerase, and the expression products are predominantly proteins important for DNA replication and late gene expression. So far, 19 viral genes have been shown to be associated with late viral gene expression, and designated "late expression factors" (LEFs) (Rapp et al., 1998; Hang et al., 1995). Nine of these genes, ie-1, ie-2, dnapol, p143, p35, lef-1, lef-2, lef-3, and lef-7, affect viral DNA replication (Iyengar et al., 1999; Vanarsdall et al., 2006). Several other genes, including lef-4, lef-5, lef-6, lef-8, lef-9, lef-10, lef-12, $p 47$, and $p p 31$, are also considered to be involved in late viral gene transcription (Lu et al., 1995).

All the sequenced baculovirus genomes include Bm67, one of the core baculovirus genes; its homologous gene in AcMNPV is ORF81 (Ge et al., 2008). The role of this protein in viral replication is as yet unclear, the understanding the biological function of Bm67, however, would clarify the mechanism of transcriptional regulation in the insect nuclear polyhedrosis viruses. Bm67 is located between genes $g p 41$ and $B m 68$, both of which are late viral genes. $g p 41$, one of the 30 core baculovirus genes, is highly conserved. During viral budding, GP41, an occlusion-derived virus membrane glycoprotein, mediates the release of the nucleocapsid from the nucleus and promotes nucleocapsid trafficking to the cell membrane (Olszewski et al., 1997). Bm68 encodes a structural protein of the budded virus (BV), and its deletion reduces the viral budding rate, leading to delayed viral DNA replication (Iwanaga et al., 2002). Although Bm67 is essential to early viral DNA replication and Bm67-deleted viruses do not form infectious virus particles (Ge et al., 2008), the role of $B m 67$ in viral gene transcription has not yet been determined.

Red recombination technology is a genetic engineering technology developed in recent years (Copeland et al., 2001). The $\lambda$ phage Red recombinase, an exogenous gene sandwiched between two sequences homologous to the target gene, undergoes homologous recombination with the target gene. This effectively inserts the foreign gene into the position of the target gene in the host genome, efficiently achieving gene knockout (Costantino et al., 2003). Red recombination technology has been widely applied to the functional analysis of the genes of AcMNPV and BmNPV (Guarino et al., 2002; Dickison et al., 2012). To investigate the function of Bm67, we generated a Bm67-deficient virus and studied the impact of this deletion on infectious virus production and viral genome replication and transcription, using wild type (encodes a complete BmNPV) and Bm67repair viruses as controls.

\section{Materials and Methods}

Cells and reagents. BmN cells, Escherichia coli strains TG1, DH10Bac and BW25113, plasmids pFastBac1 and pKD3 are all stored in our laboratory (Yu et al., 2013). 1-Arabinose was purchased from Promega (USA). SOC liquid medium was purchased from Biocolor BioScience \& Technology Company; TRIzol $^{\circledR}$ Reagent was purchased from lnvotrigen (USA). A 1-kb DNA ladder marker, DL2000 DNA ladder marker, T4 ligase, the pMD18-T vector, and restriction endonucleases BamHI, EcoRI and HindIII were purchased from TaKaRa Bio (Japan). The gel extraction kit was from Axygen (USA). Taq DNA polymerase and the corresponding PCR reactions reagents were from the Beijing Dingguo Biotechnology Company (China). KOD Plus high-fidelity DNA polymerase and the corresponding reaction reagents were purchased from Toyobo (Japan). DNA transfection reagent was purchased from SignaGen (USA). All primers were synthesized by the Shanghai Shengon Biotechnology Company (China).

Construction of the Bm67 gene-deficient Bacmid. To produce a $B m 67$ gene-deficient bacmid, a linear fragment targeting Bm67 was constructed (Fig.1a). The PCR amplification primers and all other primers sequences are shown in Table 1. A targeting fragment of around $1100 \mathrm{bp}$, designated $\mathrm{Bm} 67-\mathrm{C}$, was amplified by PCR using the pKD3 plasmid as the template and Bm67-CF and Bm67-CR as primers. It was transformed into competent $\mathrm{DH} 10 \mathrm{Bac}$ cells containing the $\mathrm{pKD} 46$ plasmid. After l-Arabinose was added to induce the expression of $\lambda$ Red recombinase, the linear fragment Bm67-C recombined with Bm67 in the viral genome (Yu et al., 2013). Positive clones were selected on plates containing chloramphenicol and kanamycin. The primers Bm67F/Bm67R and catF/catR were used to confirm the recombination and $B m 67$ deletion.

Construction of the Bm67 gene-repaired Bacmid. To generate a $B m 67$ gene-repaired bacmid, the pFastBac1-Bm67 plasmid was generated by inserting the $B m 67$ gene downstream of the polyhedrin promoter using the Bac-to-Bac ${ }^{\circledast}$ Baculovirus Expression System, as shown in Fig. 1b. The primers were based on the $B m 67$ sequence, with $B a m H I$ and EcoRI restriction sites introduced, together with $297 \mathrm{bp}$ of the Bm67 promoter sequence (Table 1). The digested PCR products were used to generate the plasmid pFastBac1-Bm67, which was transformed into DH10Bac cells containing the Bm67 gene-deficient bacmid. The positive clones were screened on plates containing Kanamycin, gentamicin, tetracycline, IPTG and 5-bromo-4-chloro-3-indolyl- $\beta$-d-galactopyranoside (X-gal). The primers for identification of the Bm67 presence were M13, pFBBm67F, and pFB-Bm67R (Table 1).

Real-time PCR assay of gp41 DNA. Real-time quantitative PCR (qPCR) was used to assess the rate of viral DNA replication. To eliminate the impact of the sample volume, the levels of $\beta$-actin were used 
Table 1. Primer sequences for PCR amplification

\begin{tabular}{|c|c|}
\hline Primer name & Primer sequence $\left(5^{\prime} \rightarrow 3^{\prime}\right)$ \\
\hline $\mathrm{Bm} 67-\mathrm{CF}^{\mathrm{a}}$ & $\begin{array}{l}\text { Forward: 5'-AAGTACACGTGTCCAATGGGTACTCGTTTGAATTTCATCCAGGCAGTCAA } \\
\text { GTGTAGGCTGGAGCTGCTTC-3' }\end{array}$ \\
\hline $\mathrm{Bm} 67-\mathrm{CR}^{\mathrm{a}}$ & $\begin{array}{l}\text { Reverse: 5'-CGGCGCAAGTCATCAGCACCGTTTGAAAACTGATGCGCCGACACAAAAT } \\
\text { GATGGGAATTAGCCATGGTCC-3' }\end{array}$ \\
\hline $\mathrm{Bm} 67 \mathrm{~F}$ & Forward: 5'-ATGACGACGACGACGACGAAGACGA-3' \\
\hline Bm67R & Reverse: 5'-TCATCTGTCATACTTTTTATTTGTA-3' \\
\hline $\begin{array}{l}\text { catF } \\
\text { catR }\end{array}$ & Forward: 5'- CACGTTTAAATCAAAACTGGTG-3' \\
\hline$\beta$-actinF & Forward: 5'-GCGCGGCTACTCGTTCACTACC-3' \\
\hline$\beta$-actinR & Reverse: 5'-TGCCGCAAGCTTCCATACCC-3' \\
\hline M13F & Forward: 5'- GTTTTCCCAGTCACGAC-3' \\
\hline M13R & Reverse: 5'-CAGGAAACAGCTATGAC-3' \\
\hline pFB-Bm67F & Forward: 5'-CGCGGATCCAATATTACTTAAAAAAACGGGGCGT-3' \\
\hline pFB-Bm67R & Reverse: 5'-CCGGAATTCTCATCTGTCATACTTTTTATTTGTA-3' \\
\hline gp41F & Forward: 5'-CGTAGTGGTAGTAATCGCCGC-3' \\
\hline gp41R & Reverse:5'-AGTCGAGTCGCGTCGCTTT-3' \\
\hline Bmie-1F & Forward: 5'-CGAGACGGCTGCACAAAA-3' \\
\hline Bmie-1R & Reverse: 5'-TGCCCAAAAGAAACCCACA-3' \\
\hline Bmlef-3F & Forward: 5'-TCGGATGACCGTTCTACCTCTT-3' \\
\hline Bmlef-3R & Reverse: 5'-CTTCCAGCAGCATTGAGATTTG-3' \\
\hline Bmvp39F & Forward: 5'-AGACACCACAAACCCGAACAC-3' \\
\hline Bmvp39R & Reverse: 5'-TTGATCGCCAACACCACCT-3' \\
\hline BmvP10F & Forward: 5'-TTGATCGCCAACACCACCT-3' \\
\hline BmvP10R & Reverse: 5'-CGATTCTTCCAGCCCGTTT-3' \\
\hline BmdnapolF & Forward: 5'-GCCGATTTGCGTTTTTTGCC-3' \\
\hline BmdnapolR & Reverse: 5'-GATTGCCATTTGTGCTTGTT-3' \\
\hline
\end{tabular}

These two primers were composed of 50-bp Bm67 homology arms (underlined) and a 20-bp cat homologous zone. Letters in boxes represent the restriction enzymes BamH' and EcoR'.

as an internal control, amplified with primers $\beta$-actinF and $\beta$-actinR. $\mathrm{BmN}$ cells in the logarithmic growth phase $\left(1 \times 10^{6} / 35 \mathrm{~mm}\right.$ dish $)$ were transfected with $1 \mu \mathrm{g}$ of Bm67-KO-Bacmid, the wild-type bacmid (wtBacmid), or Bm67-Re-Bacmid DNA using DNA transfection reagent (SignaGen) according to the manufacturer's instructions, and the cells were harvested $6,12,24,36$, and $48 \mathrm{hr}$ after transfection. Total DNA was extracted, digested with $D p n \mathrm{I}$ for $5 \mathrm{hr}$ to remove any exogenous methylated bacmid DNA, and analyzed with qPCR using the $g p 41$ gene-specific primers gp $41 \mathrm{~F}$ and gp41R (Vanarsdall et al., 2005).

Real-time RT-PCR assay of transcripts of the dnapol, ie-1, lef-3, $v p 39$ and p10 genes. The timing and extent of viral gene transcription was assessed with qPCR. BmN cells were transfected with bacmids as described above and their DNA was harvested 6, 12, 24, 36 , and $48 \mathrm{hr}$ later. Total RNA was extracted with TRIzol ${ }^{\circ}$ Reagent and treated with DNaseI to remove any residual BmNPV genomic DNA. cDNA was synthesized with reverse transcriptase and an oligo $(\mathrm{dT})_{18}$ primer and amplified with BmNPV ie-1, lef-3, vp39, dnapol, or $p 10$ gene-specific primers. The primer pairs Bmie-1F/ Bmie-1R, Bmlef-3F/Bmlef-3R, Bmvp39F/Bmvp39R, BmP10F/ $\mathrm{BmP10R}$, and BmdnapolF/BmdnapolR were used (Table 1). $\beta$-Actin was amplified as the control, as described above.

Virus titration. BmN cells were transfected with bacmids as described above. Cell culture supernatants were collected 12, 18, 24,
48,72 , and $96 \mathrm{hr}$ after transfection, and the $50 \%$ tissue culture infectious dose $\left(\mathrm{TCID}_{50}\right)$ was calculated. BmN cells in the logarithmic growth phase were seeded in 96 -well plates at $1 \times 10^{3}$ cells / well. The viral supernatant was diluted and loaded into each well, and the cells were cultured for five days. The viral titer $\left(\mathrm{TCID}_{50}\right)$ was calculated with the method of Reed and Muench (Reed et al., 1938).

Electron microscopy. BmN cells $\left(1 \times 10^{5} / 35 \mathrm{~mm}\right.$ dish $)$ were transfected with $1 \mu \mathrm{g}$ of Bm67-KO-Bacmid, wtBacmid, or Bm67Re-Bacmid DNA, and the cells were collected $24 \mathrm{hr}$ after transfection. The cells were fixed in $2.5 \%$ glutaraldehyde solution at $4{ }^{\circ} \mathrm{C}$ overnight and processed according to the standard procedures. $70-90 \mathrm{~nm}$ thin sections were cut and observed and analyzed with a transmission electron microscope (HitachiH-7650).

\section{Results}

\section{Construction of Bm67-deficient and Bm67-repaired} bacmids

Following the recombination in E.coli to delete the Bm67 gene by replacement with a linear fragment Bm67-C cas- 
sette, a single clone was randomly selected from agar plates containing antibiotics and cultured overnight. Bacmid DNA was confirmed with PCR using wtBacmid as the control and the following primer pairs: $\mathrm{Bm} 67 \mathrm{~F} / \mathrm{catR}$, catF/Bm67R, and Bm67F/Bm67R. As shown in Fig. 2a, although no band was detected in the control wtBacmid-transfected cells (data not shown), the sizes of the amplification products were about $660 \mathrm{bp}, 990 \mathrm{bp}$, and $1600 \mathrm{bp}$ when primer pairs Bm67F/catR, $\mathrm{Bm} 67 \mathrm{R} / \mathrm{catF}$, and $\mathrm{Bm} 67 \mathrm{~F} / \mathrm{Bm} 67 \mathrm{R}$ were used, respectively. These results suggest that the $B m 67$ gene was successfully deleted from the bacmid genome, and this virus was designated "Bm67-KO-Bacmid".

Following recombination to repair the Bm67-KO-Bacmid and re-introduce the $B m 67$ gene, a single clone was randomly selected from the agar plates containing antibiotics, and cultured overnight. The bacmid DNA was confirmed with PCR using the following primer pairs: M13F/M13R, $\mathrm{M} 13 \mathrm{~F} / \mathrm{pFB}-\mathrm{Bm} 67 \mathrm{R}$, and pFB-Bm67F/M13R. As shown in Fig. $2 \mathrm{~b}$, the sizes of the amplification products when primer pairs M13F/M13R, M13F/pFB-Bm67R, or pFB-Bm67F/ $\mathrm{M} 13 \mathrm{R}$ were used were roughly $3,700 \mathrm{bp}, 2,500 \mathrm{bp}$, and 1,400 $\mathrm{bp}$, respectively, which is consistent with the calculated size, suggesting that the $B m 67$ gene was successfully repaired. This virus was designated "Bm67-Re-Bacmid".

\section{Effect of Bm67 gene deletion on replication of viral} genomic DNA

To analyze the effects of $B m 67$ gene deletion on BmNPV DNA replication, BmN cells were transfected with Bm67KO-Bacmid, wtBacmid, or Bm67-Re-Bacmid DNA, and the cells were harvested $6,12,24,36$, and $48 \mathrm{hr}$ after transfection. As illustrated in Fig. 3, the viral DNA copy number in the Bm67-KO-Bacmid-transfected cells was lower at $12 \mathrm{hr}$ than in cells transfected with wtBacmid or Bm67-Re-Bacmid $(P<0.05)$ and significantly lower at $24-48 \mathrm{hr}(P<0.01)$. The viral DNA copy numbers in the wtBacmid- or Bm67-ReBacmid-transfected cells increased significantly between 24 and $48 \mathrm{hr}$ post transfection (p.t.). In contrast, although the viral DNA copy number in cells transfected with the Bm67-deletion virus also increased during this period, it was significantly lower than in control cells, suggesting that the BmNPV Bm67 gene is essential for genome replication.

$$
\text { Effec }
$$

Effect of Bm67 gene deletion on infectious virus replica-

The supernatants of BmN cells transfected with Bm67KO-Bacmid, wtBacmid, or Bm67-Re-Bacmid were collected $12,18,24,48,72$, and 96 hr p.t., and the viral titers determined (Fig. 4). The supernatant of the cells transfected with Bm67-KO-Bacmid was not infectious, indicating that $\mathrm{Bm} 67-\mathrm{KO}-\mathrm{Bacmid}$ did not produce infectious viral particles. (a)

(b)

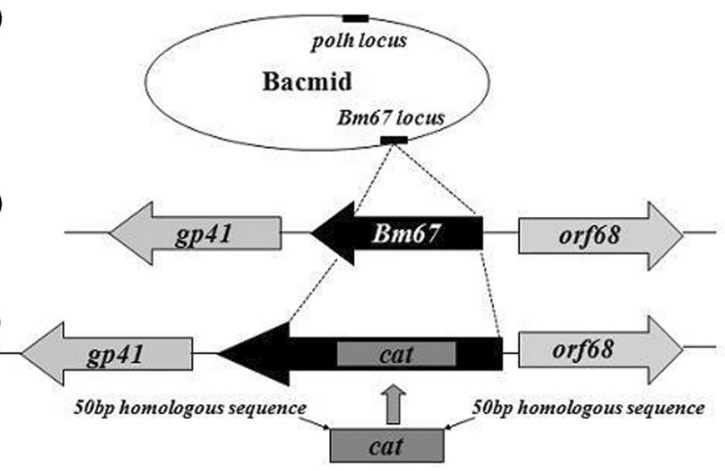

(d)

(e)

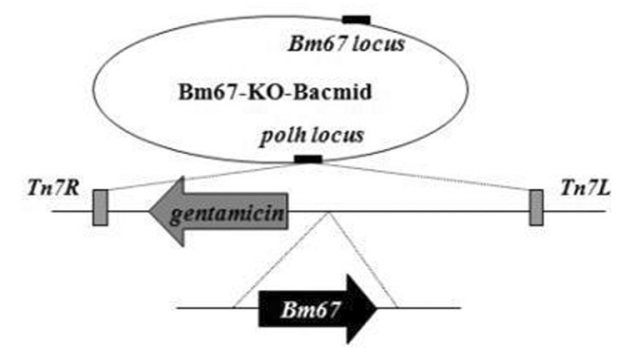

Fig. 1

Construction of Bm67-deficient and Bm67-repaired bacmids (a) Location of Bm67 in the Bm67-KO-Bacmid genome; (b) recombination between cat and Bm67; (c) PCR amplification of the target fragment using the $\mathrm{pKD} 3$ plasmid as the template and primers Bm67-CF/Bm67-CR; (d) location of polh in the Bm67-Re-Bacmid genome; (e) insertation of Bm67, with its promoter, into the transposon; (f) PCR amplification of Bm67 and its promoter using the wild-type bacmid as the template.
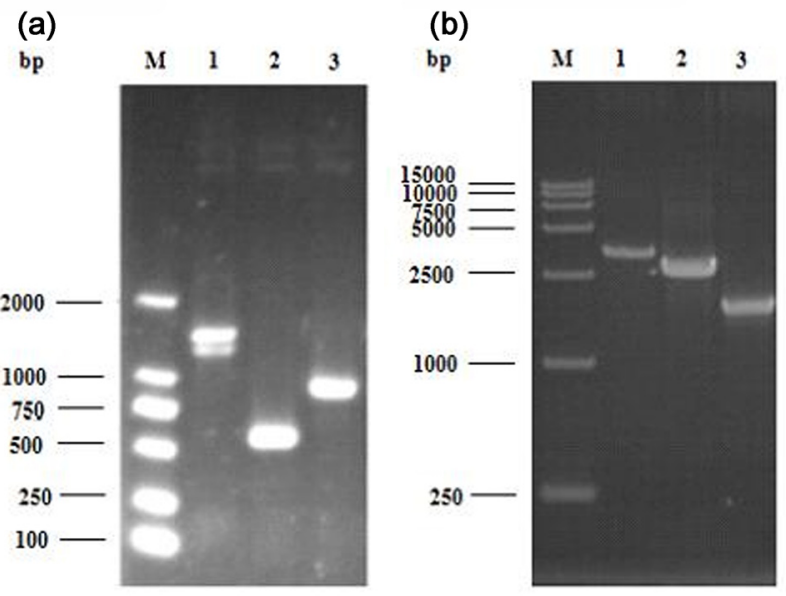

Fig. 2

Validation of Bm67-deficient (a) and Bm67-repaired viruses (b) by PCR

Lane 1: primers Bm67F and Bm67R were employed; lane 2: primers Bm67F and cat R were employed; lane 3: primers Bm67R and catF were employed; lane 4: The primers M13F and M13R were employed; lane 5: primers M13F and $\mathrm{pFbBm} 67 \mathrm{R}$ were employed; lane 6: primers $\mathrm{pFbBm67F}$ and M13R were employed. 


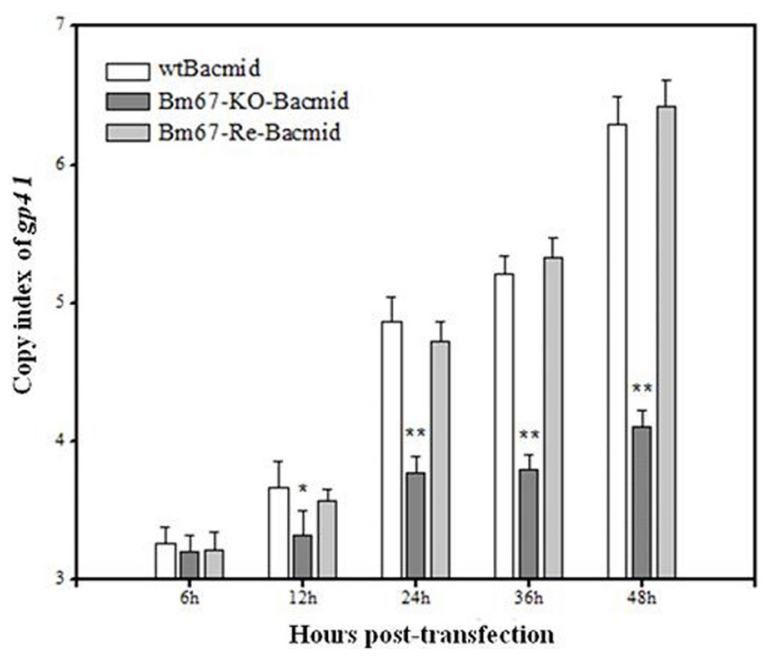

Fig. 3

Virus copy number in cells transfected with bacmids

The cells transfected with the bacmids were harvested at $6-48 \mathrm{hr}$ p.t. and assayed for $g p 41$ by real-time PCR. ${ }^{*} P<0.05,{ }^{* *} P<0.01$ vs wtBacmid and Bm67-Re-Bacmid. Values are expressed as means \pm SEM. Similar results were obtained in three independent experiments. $\mathrm{P}$ value was calculated by SPSS software, comparing the average copy level of Bm67-KO-Bacmid against the average copy levels of wtBacmid and Bm67-Re-Bacmid.

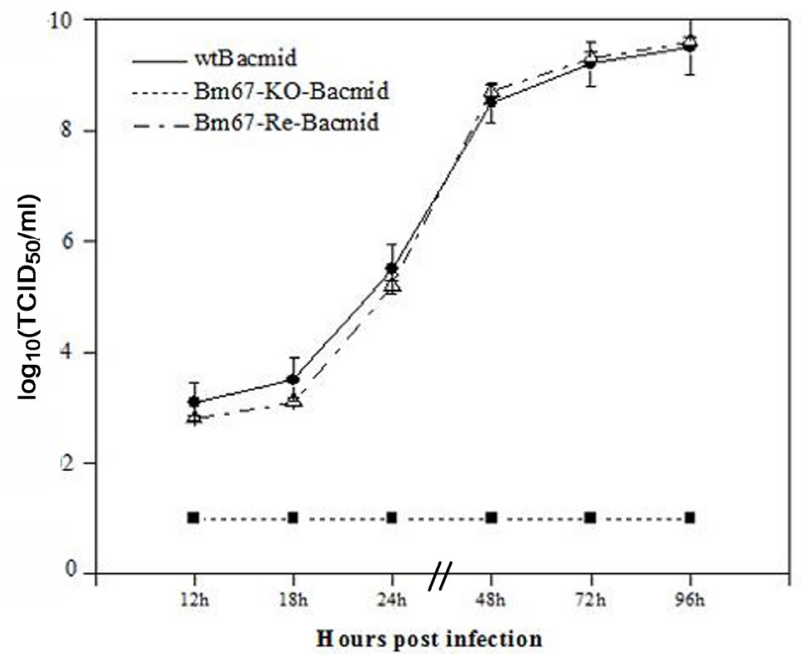

Fig. 4

Virus titers in cells transfected with bacmids

The cells transfected with the bacmids were harvested at at 6- $48 \mathrm{hr}$ p.t. and assayed for the virus titers. (a)

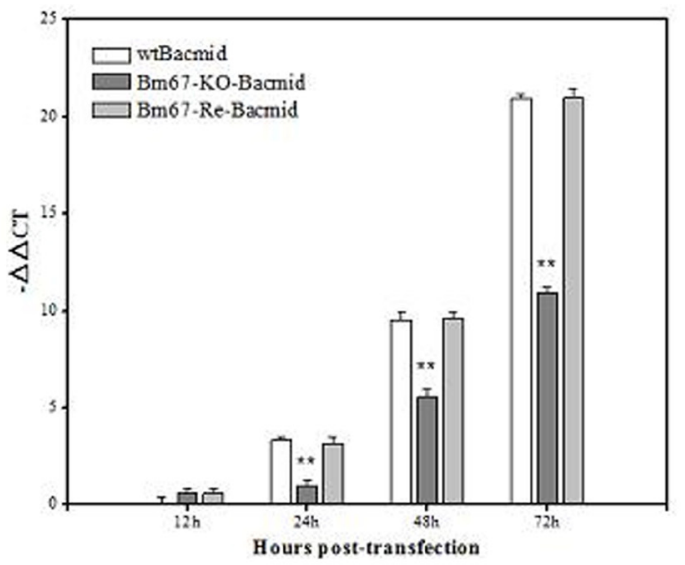

(b)

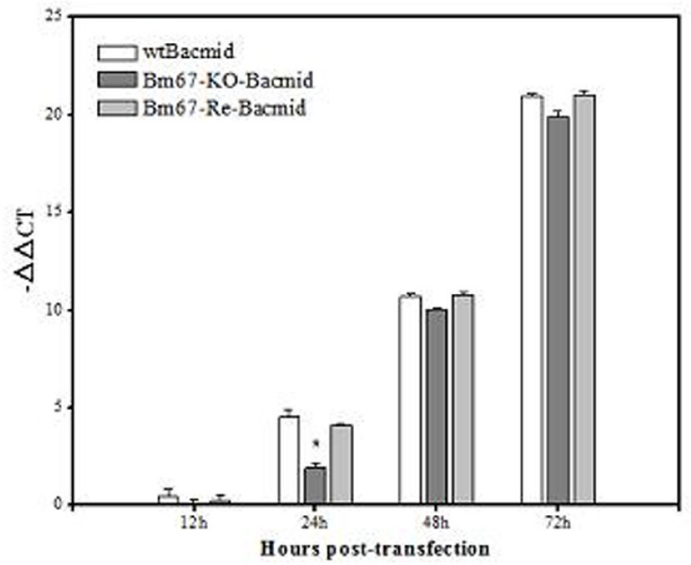

(c)

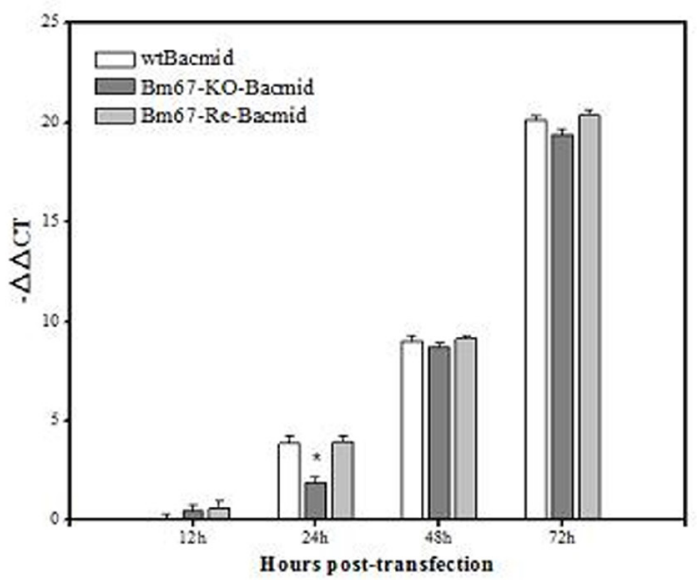

Fig. 5

Levels of transcripts of early genes dnapol (a), ie-1 (b) and lef-3 (c) in cells transfected with bacmids

Real-time RT-PCR was employed. ${ }^{\star} P<0.05$ vs wtBacmid and Bm67Re-Bacmid. Values are expressed as means \pm SEM. Similar results were obtained in three independent experiments. $\mathrm{P}$ value was calculated by SPSS software, comparing the average transcriptional copy level of Bm67KO-Bacmid against the average transcriptional copy levels of wtBacmid and Bm67-Re-Bacmid. 
The supernatant of the cells transfected with wtBacmid or Bm67-Re-Bacmid yielded infectious virus, and the viral titers increased rapidly, almost reaching their maxima at $48 \mathrm{hr}$.

\section{Effect of Bm67 gene deletion on viral gene transcription}

Because gene expression in BmNPV is mainly regulated at the transcriptional level, early viral genes can be transcribed by the host RNA polymerase, whereas the late viral genes can be transcribed by viral RNA polymerase (Todd et al., 1996). To study the effects of Bm67 deletion on BmNPV gene transcription, we measured the levels of several early genes essential to viral replication. Three early genes, dnapol, $i e-1$, and lef-3, were analyzed in cells transfected with Bm67KO-Bacmid, wtBacmid, or Bm67-Re-Bacmid. As shown in Fig. 5, the level of dnapol transcripts in cells transfected with Bm67-KO-Bacmid was only $10 \%$ of that detected in the wtBacmid- and Bm67-Re-Bacmid-transfected cells $24 \mathrm{hr}$ after transfection, indicating a significant reduction in dnapol expression. Although the transcription of dnapol in cells transfected with Bm67-KO-Bacmid had increased at 48 and $72 \mathrm{hr}$ p.t., it was always significantly lower than that detected in the wtBacmid- and Bm67-Re-Bacmid-transfected cells $(P<0.01)$. Similarly, $24 \mathrm{hr}$ after transfection, the transcription of $i e-1$ and lef- 3 in cells transfected with Bm67-KO-Bacmid were $40 \%$ and $45 \%$, respectively, of that detected in cells transfected with Bm67-Re-Bacmid or wtBacmid $(P<0.05)$. The transcription levels of $i e-1$ and $l e f-3$ in cells transfected with Bm67-KO-Bacmid had increased 48 and $72 \mathrm{hr}$ p.t., and were only slightly lower than those detected in the wtBacmid- and Bm67-Re-Bacmid-transfected cells $(P>0.05)$.

To determine the impact of $B m 67$ on the transcription of late and very late genes, we analyzed the expression of the late gene $v p 39$ and very late gene $p 10$ in cells transfected with Bm67-KO-Bacmid, wtBacmid, or Bm67-Re-Bacmid. As shown in Fig. 6, the timing and levels of transcription of the late gene $v p 39$ and the very late gene $p 10$ in the Bm67KO-Bacmid-transfected cells did not differ significantly from those in the cells transfected with wtBacmid or Bm67-ReBacmid $(P>0.05)$.

\section{Effect of Bm67 gene deletion on virus assembly}

To analyze the impact of $B m 67$ deletion on virus particle assembly, ultrathin sections were generated from wtBacmid-, Bm67-KO-Bacmid-, and Bm67-Re-Bacmid-transfected $\mathrm{BmN}$ cells and observed with a transmission electron microscope. As shown in Fig. 7, there were no viral particles in the Bm67-KO-Bacmid-transfected cell nuclei, suggesting that the loss of Bm67 affected virus assembly. However, the wtBacmid- and Bm67-Re-Bacmid-transfected cells showed the typical symptoms of baculovirus infection, including the enlarged nuclei and the formation in the cell nucleoli (a)

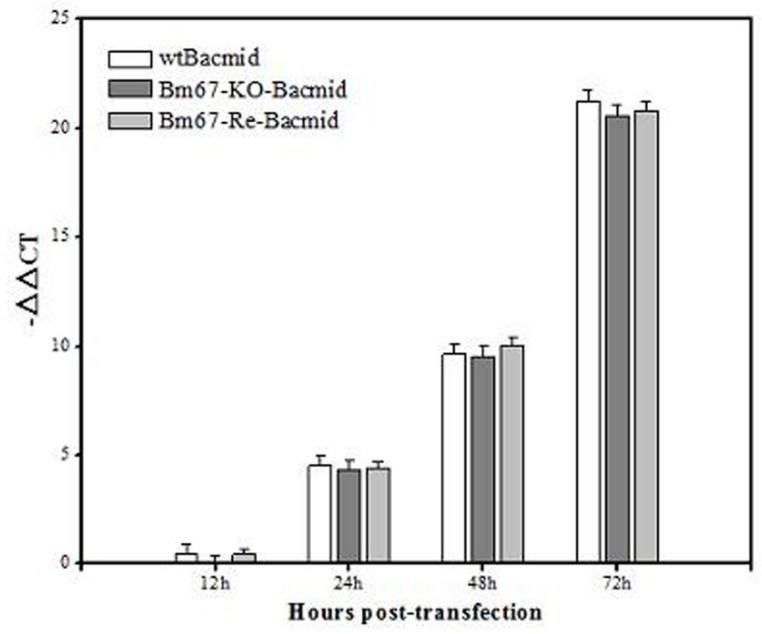

(b)

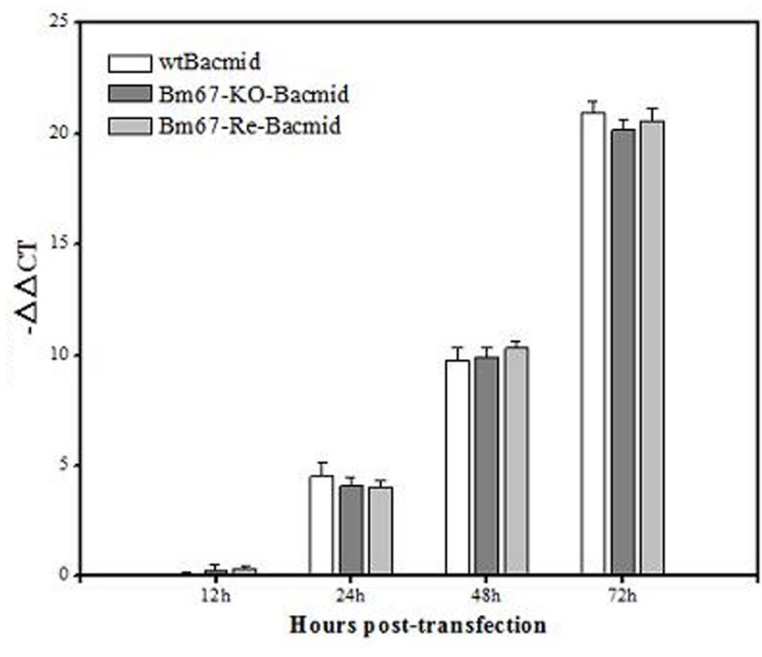

Fig. 6

Levels of transcripts of late genes $v p 39$ (a) and $p 10$ (b) in cells transfected with bacmids

Real-time RT-PCR was employed. Values are expressed as means \pm SEM. Similar results were obtained in three independent experiments.

of virogenic stroma, and a large number of nucleocapsids were observed within the nuclei. This is consistent with the measurements of viral titers.

\section{Discussion}

In this study, the Red recombination technology was used to generate a Bm67-deletion virus, Bm67-KO-Bacmid. To construct a Bm67-repaired virus, Bm67-Re-Bacmid, the $B m 67$ gene and promoter were inserted downstream from the polyhedrin promoter in Bm67-KO-Bacmid using the 


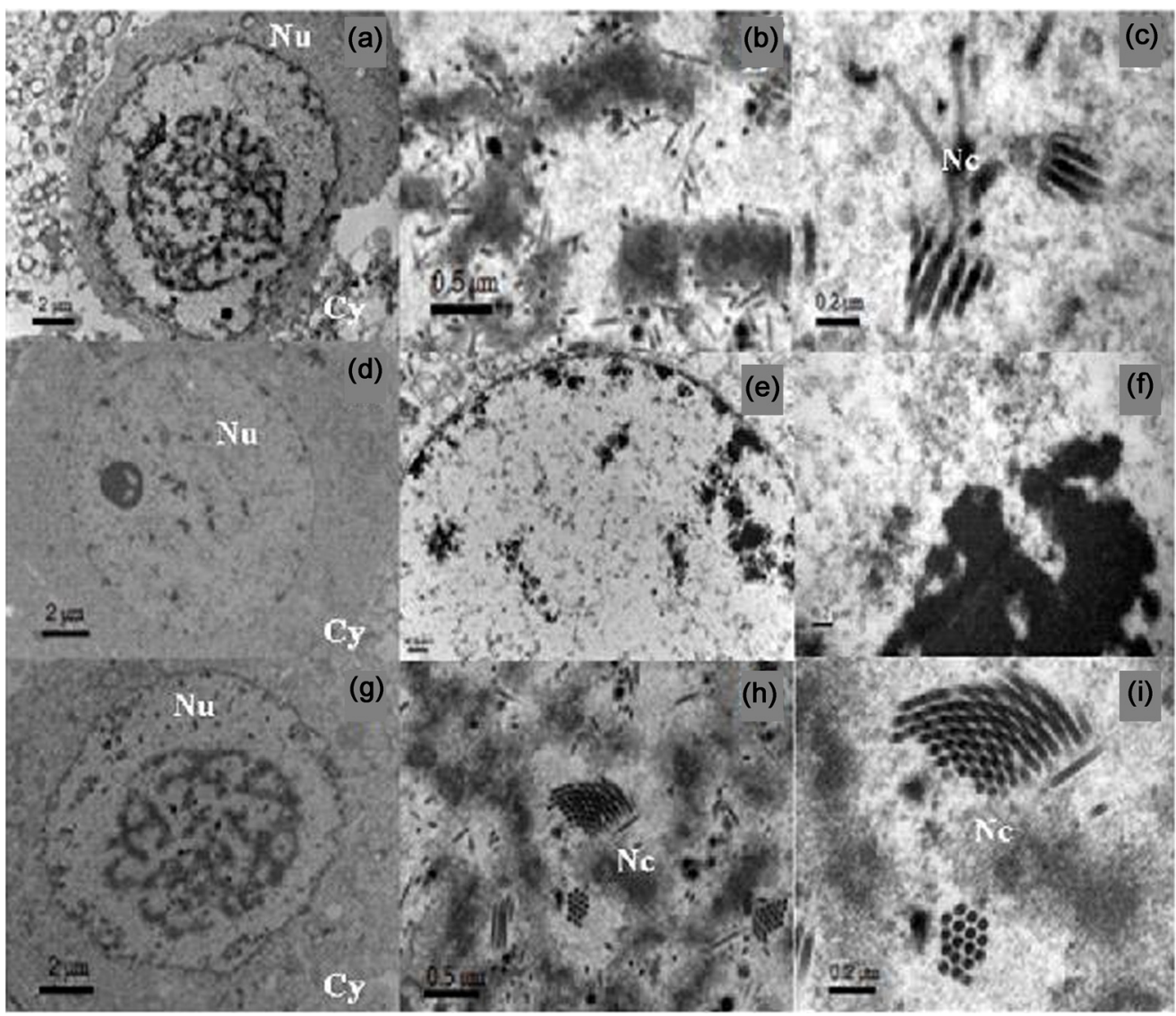

Fig. 7

Electron microscopic analysis of cells transfected with the bacmids

Cells analyzed $24 \mathrm{hr}$ p.t. wtBacmid: magnification 5,000 $\times$ (a), 20,000 $\times$ (b) and 50,000 $\times$ (c) ; Bm67-KO-Bacmid: magnification 5,000 $\times($ d) $, 20,000 \times($ e) and 50,000 × (f); Bm67-Re-Bacmid: magnification 5,000 × (g), 20,000 $\times(\mathrm{h})$ and 50,000 × (i). Nc, nucleocapsid; Nu, nucleus; Cy, cytoplasm. Bars indicate the scale.

Bac-to-Bac ${ }^{\circledast}$ Baculovirus Expression System. BmN cells transfected with the Bm67-deleted virus did not produce infectious viral particles. However, this phenotype was rescued by the ectopic expression of the Bm67 gene, suggesting that this gene is critical for the formation of infectious viral particles. These findings are consistent with those published by Ge et al. (2008) who demonstrated that the deletion of $B m 67$ reduces viral nucleocapsid assembly, and hinders the formation of BV in enveloped viruses. Our transmission electron microscopy results also confirmed that $B m 67$ deletion affects the capacity for virus assembly, leading to a failure to form mature viral particles. However, the Bm67restored virus, Bm67-Re-Bacmid, formed a large number of nucleocapsids in the nuclei.

Many processes may be inhibited in the Bm67-deficient virus, including viral DNA replication, the regulation of gene expression, and nucleocapsid assembly and transport (Oomens and Blissard, 1999; Guarino et al., 2002; Milks et al., 2003; Yamagishi et al., 2007; Ke et al., 2008). We found that the level of viral DNA replication was significantly lower in the Bm67-KO-Bacmid-transfected cells than in the cells transfected with wtBacmid or Bm67-Re-Bacmid, and that the cells transfected with Bm67-KO-Bacmid yielded supernatant with low infectivity. We concluded that although $B m 67$ enhances BV assembly, the Bm67-deficient viral genome can still replicate, albeit at a slower rate. Because BV are usually produced following the second round of infection (Volkman, 2007), 24hr after transfection, the Bm67-KOBacmid may allow only one round of replication, leading to a limited increase in the viral genome copy number, but few infectious BV.

Previous studies have highlighted the association between certain specific replication factors and baculovirus replication (Lu et al., 1995; Yu et al., 2013; Vanarsdall et al., 2004). For example, Vanarsdal et al. found that the knockout of $v l f-1$, which is expressed at the late stage of replication, inhibits viral DNA replication and prevents BV production and dissemination (Vanarsdall et al., 2004). Yu et al. (2013) deleted 
the late-expression factor lef-10 and observed reduced viral DNA replication and the reduced transcription of early and late genes. Therefore, we hypothesized that $B m 67$ may reduce the transcription levels of some replication-regulating factors. We investigated the transcription levels of several viral genes in cells transfected with the Bm67-deficient virus, and found that $B m 67$ deletion affected the transcription levels of the early genes ie-1, lef-3, and dnapol to different degrees. Twenty-four hours after the cells were transfected with Bm67-KO-Bacmid, the transcript level of the dnapol genes was only $10 \%$ of that in cells transfected with wtBacmid or Bm67-Re-Bacmid. Similarly, the transcription levels of ie-1 and lef-3 were only $40 \%$ and $45 \%$, respectively, of those in cells transfected with wtBacmid or Bm67-Re-Bacmid. In contrast, the transcription of the late gene $v p 39$ and the very late gene $p 10$ was barely affected by the deletion of $B m 67$. These findings suggest that $B m 67$ deletion reduces the expression of the viral DNA polymerase gene (dnapol) and other early genes, which are required for viral DNA replication, and consequently limits viral genome replication (Okano et al., 1999). Interestingly, the levels of ie-1 and lef-3 transcripts in the cells transfected with Bm67-KO-Bacmid had increased significantly $48 \mathrm{hr}$ after transfection, and were restored to the levels detected in cells transfected with the wild-type virus. Therefore, we hypothesize that the deletion of Bm67 only delayed the transcription of some early genes. In contrast, dnapol transcription was depressed at all the time points tested. The baculovirus-specific DNA polymerase, originally identified by Mikhailov et al. in BmNPV, has a $3^{\prime} \rightarrow 5^{\prime}$ exonuclease activity and is involved in the synthesis of the DNA leading strand and lagging strand, thus ensuring the fidelity of DNA synthesis (Mikhailov et al., 1986). In 2005, Vanarsdall et al. found that the replication of the viral genomic DNA is inhibited by the knockout of dnapol (Vanarsdall et al., 2005).

In summary, we speculate that the reduced levels of viral DNA replication that result from $\mathrm{Bm} 67$ deletion are caused by the reduced transcription of viral dnapol and the delayed transcription of some early genes. A further in-depth study is required to determine the influence of $B m 67$ deletion on the expression levels of viral early genes.

Acknowledgements. This work was supported by the grants No. 2011AA100603 from National High-tech R\&D program (863 Program) and No. 31101831 from National Natural Science Foundation of China.

\section{References}

Ayres MD, Howard SC, Kuzio J, Lopez-Ferber M, Possee RD (1994): The complete DNA sequence of autographa californica nuclear polyhedrosis virus. Virology 202, 586-605. http:// dx.doi.org/10.1006/viro.1994.1380

Bulach DM, Kumar CA, Zaia A, Liang B, Tribe DE (1999): Group II nucleopolyhedrovirus subgroups revealed by phylogenetic analysis of polyhedrin and DNA polymerase gene sequences. J. Invertebr. Pathol. 73, 59-73. http://dx.doi. org/10.1006/jipa.1998.4797

Copeland NG, Jenkins NA, Court DL (2001): Recombineering: a powerful new tool for mouse functional genomics. Nat. Rev. Genet. 2, 769-779. http://dx.doi. org $/ 10.1038 / 35093556$

Costantino N, Court DL (2003): Enhanced levels of $\lambda$ Red-mediated recombinants in mismatch repair mutants. Proc. Natl. Acad. Sci. USA 100, 15748-15753. http://dx.doi. org/10.1073/pnas.2434959100

Dickison VL, Willis LG, Sokal NR, Theilmann DA (2012): Deletion of AcMNPV ac146 eliminates the production of budded virus. Virology 431, 29-39. http://dx.doi.org/10.1016/j. virol.2012.05.002

Ge JQ, Yang ZN, Tang XD, Xu HJ, Hong J, Chen JG, Zhang CX (2008): Characterization of a nucleopolyhedrovirus with a deletion of the baculovirus core gene Bm67. J. Gen. Virol. 89, 766-774. http://dx.doi.org/10.1099/vir.0.83398-0

Gomi S, Majima K, Maeda S (1999): Sequence analysis of the genome of Bombyx mori nucleopolyhedrovirus. J. Gen. Virol. 80, 1323-1337.

Guarino LA, Mistretta TA, Dong W (2002): Baculovirus lef-12 is not required for viral replication. J. Virol. 76, 12032-12043. http://dx.doi.org/10.1128/JVI.76.23.12032-12043.2002

Hang X, Dong W, Guarino LA (1995): The lef-3 gene of Autographa californica nuclear polyhedrosis virus encodes a singlestranded DNA-binding protein. J. Virol. 69, 3924-3928.

Iyengar B, Roote J, Campos AR (1999): The tamas gene, identified as a mutation that disrupts larval behavior in Drosophila melanogaster, codes for the mitochondrial DNA polymerase catalytic subunit (DNApol- $\gamma 125)$. Genetics $153,1809-1824$.

Iwanaga M, Kurihara M, Kobayashi M, Kang W (2002): Characterization of Bombyx mori nucleopolyhedrovirus orf68 gene that encodes a novel structural protein of budded virus. Virology 297, 39-47. http://dx.doi.org/10.1006/ viro.2002.1443

Ke J, Wang J, Deng R, Wang X (2008): Autographa californica multiple nucleopolyhedrovirus ac66 is required for the efficient egress of nucleocapsids from the nucleus, general synthesis of preoccluded virions and occlusion body formation. Virology 374, 421-431. http://dx.doi. org/10.1016/j.virol.2007.12.033

Lu A, Miller LK (1995): The roles of eighteen baculovirus late expression factor genes in transcription and DNA replication. J. Virol. 69, 975-982.

Mikhailov VS, Marlyev KA, Ataeva JO, Kullyev PK, Atrazhev AM (1986): Characterization of $3^{\prime} \rightarrow 5^{\prime}$ exonuclease associated with DNA polymerase of silkworm nuclear polyhedrosis virus. Nucl. Acids. Res. 14, 3841-3857. http://dx.doi. org/10.1093/nar/14.9.3841

Milks ML, Washburn JO, Willis LG, Volkman LE, Theilmann DA (2003): Deletion of pe38 attenuates AcMNPV genome 
replication, budded virus production, and virulence in heliothis virescens. Virology 310, 224-234. http://dx.doi. org/10.1016/S0042-6822(03)00143-0

Okano K, Mikhailov VS, Maeda S (1999): Colocalization of baculovirus IE- 1 and two DNA-binding proteins, DBP and LEF-3, to viral replication factories. J. Virol. 73, 110-119.

Olszewski J, Miller LK (1997): A role for baculovirus GP41 in budded virus production. Virology 233, 292-301. http:// dx.doi.org/10.1006/viro.1997.8612

Oomens AG, Blissard GW (1999): Requirement for GP64 to drive efficient budding of Autographa californica multicapsid nucleopolyhedrovirus. Virology 254, 297-314. http:// dx.doi.org/10.1006/viro.1998.9523

Passarelli AL, Guarino LA (2007): Baculovirus late and very late gene regulation. Curr. Drug Targets 8, 1103-1115. http:// dx.doi.org/10.2174/138945007782151324

Rapp JC, Wilson JA, Miller LK (1998): Nineteen baculovirus open reading frames, including LEF-12, support late gene expression. J. Virol. 72, 10197-10206.

Reed LJ, Muench H (1938): A simple method of estimating fifty percent endpoints. Am. J. Epidemiol. 27, 493-497.

Todd JW, Passarelli AL, Lu A, Miller LK (1996): Factors regulating baculovirus late and very late gene expression in transientexpression assays. J. Virol. 70, 2307-2317.
Vanarsdall AL, Okano K, Rohrmann GF (2005): Characterization of the replication of a baculovirus mutant lacking the DNA polymerase gene. Virology 331, 175-180. http://dx.doi. org/10.1016/j.virol.2004.10.024

Vanarsdall AL, Okano K, Rohrmann GF (2006): Characterization of the role of very late expression factor 1 in baculovirus capsid structure and DNA processing. J. Virol. 80, 1724-1733. http://dx.doi.org/10.1128/JVI.80.4.1724-1733.2006

Vanarsdall AL, Okano K, Rohrmann GF (2004): Characterization of a baculovirus with a deletion of vlf-1. Virology 326, 191-201. http://dx.doi.org/10.1016/j.virol.2004.06.003

Volkman LE (2007): Baculovirus infectivity and the actin cytoskeleton. Curr. Drug Targets 8, 1075-1083. http://dx.doi. org/10.2174/138945007782151379

Yamagishi J, Burnett ED, Harwood SH, Blissard GW (2007): The AcMNPV pp31 gene is not essential for productive AcMNPV replication or late gene transcription but appears to increase levels of most viral transcripts. Virology 365, 34-47. http://dx.doi.org/10.1016/j.virol.2007.02.034

Yu W, Du CY, Quan YP, Nie ZM, Chen J, Lv ZB, Zhang YZ (2013): Characterization of late gene expression factor LEF-10 from Bombyx mori nucleopolyhedrovirus. Virus Res. 175, 45-51. http://dx.doi.org/10.1016/j. virusres.2013.03.022 